\title{
Reaching strategies of very preterm infants at 8 months corrected age
}

\author{
Helena Grönqvist · Katarina Strand Brodd • \\ Claes von Hofsten
}

Received: 26 July 2010/Accepted: 29 December 2010/Published online: 26 January 2011

(C) The Author(s) 2011. This article is published with open access at Springerlink.com

\begin{abstract}
Reaching strategies and kinematics for a group of very preterm infants were investigated and compared with a group of full-term infants when reaching for a moving object. Eight-month-old (corrected-age) infants were presented with small toys moving on a semicircular path in the vertical plane. The trajectories of the target and the hands of the infants were measured using a 3D motion analysis system. No differences were found in how often the infants encountered the target. The very preterm group, however, used bimanual strategies more often and had more curved reaching paths than the full-term group. These results suggest that very preterm infants are equally successful as healthy full-term infants in catching a moving object but their reaching strategies are less efficient compared with full-term infants at 8 months (corrected age).
\end{abstract}

Keywords Bimanual - Development - Moving objects . Preterm infants $\cdot$ Reaching $\cdot$ Kinematics

\section{Introduction}

The mastering of reaching and grasping is one of the most important developmental accomplishments during the first year of life. At around 4 months, infants begin to successfully grasp objects positioned within reach in front of

H. Grönqvist $(\bowtie) \cdot$ C. von Hofsten

Department of Psychology, Uppsala University,

Box 122575142 Uppsala, Sweden

e-mail: Helena.gronqvist@psyk.uu.se

K. Strand Brodd

Department of Women's and Children's Health,

Uppsala University, Uppsala, Sweden them. At the same age, they also catch moving objects by anticipating their future positions (von Hofsten 1980; von Hofsten 1983). Research on full-term (FT) infants has shown that the use of uni- and bimanual strategies varies during the first year of life (Corbetta and Thelen 1996). When reaching out with one hand, the point of gravity shifts and causes obstruction of balance that can be compensated by reaching out with the other hand as well. Thus, using both hands may provide the infants with better postural stability during reaching (Rochat 1992). Kinematic properties (e.g., velocity, movement units, and relative length) are used to analyze the efficiency and development of the hand movements. Healthy FT infants catch objects moving with at least $30 \mathrm{~cm} / \mathrm{s}$ at 18 weeks of age (von Hofsten 1980) and 8-month-olds catch objects moving with $120 \mathrm{~cm} / \mathrm{s}$ (von Hofsten 1983). When the object moves horizontally in front of the infants, they aim the reach at a point where the object is going to be caught and not for the point where the object is at the beginning of the reach.

How is this skill affected by prematurity? The reaches for stationary objects by infants born preterm (PT) have somewhat different kinematic properties than those of FT infants. Toledo and Tudella (2008) found that the average and final velocities were slower and more adjustments were made compared with full terms. The more pronounced this tendency was for the PT infants, the higher was the rate of successful reaches. Fallang et al. (2003) measured kinematic movement quality of reaches for stationary objects in terms of a combined measure of peak velocity and number of movement units in a group of low-risk and high-risk PT infants. High risk was defined as infants having an Apgar score of 3 or less at $5 \mathrm{~min}$ and/or respiratory problems requiring ventilator support. At 4 months corrected age (CA), it was found that the low-risk preterm infants 
displayed better reaching qualities than the full-term control group and the high-risk PT infants. This advantage, however, had disappeared by 6 months and the high-risk infants began to show poorer reaching behavior than the other two groups. A follow-up study at 6 years showed that the preterm infants who did not reach for the object at 4 months CA were at high risk for developing minor neurological dysfunction but this relationship did not exist for the FT infants who at 4-month-olds did not reach at all (Fallang et al. 2003; Fallang et al. 2005). Thus, the results indicate that at around the onset of functional reaching, the longer extrauterine experience of low-risk PT infants actually gives them a temporary advantage relative to FT, at least during the first half-year of year of life. The highrisk PT infants, however, were always at a developmental disadvantage.

There are a number of possible disturbances of brain development that might have contributed to the deficient reaching behavior in the premature infants studied. However, one of the leading causes of brain injury in infants born preterm is periventricular leukomalacia (PVL), i.e., hypoxic lesions to the afferent pathways of the white matter (Volpe 2001). These lesions are often located in the posterior regions of the white matter, regions that connect thalamus with the visual cortex, i.e., the magno-cellular pathways. These pathways continue into the dorsal pathways that are involved in sensory-motor coordination (Goodale and Milner 1992; Atkinson 2000). The damages can affect various perceptual and motor functions, e.g., the ability to identify and interpret motion. PVL is the leading cause of motor disability in preterm children (Hoon et al. 2002). In addition, children with PVL can have structurally normal eyes but still have visual field defects, nystagmus, reduced visual acuity, and difficulties organizing their perceptions (Jacobson et al. 1996).

Most research on reaching in the PT population has considered reaching for stationary objects. In order to reach successfully for a moving ones, infants must be able to perceive and evaluate its motion. Kayed and van der Meer (2009) studied 5 PT infants longitudinally from 22 to 48 weeks corrected age and found that one of the infants showed a deviant catching development relative to FT infants. Infants typically used a less efficient velocity strategy at younger ages and switch into a more efficient time strategy sometime between 30 and 48 week, but this infant did not make that shift.

If the magno-cellular or dorsal pathways are damaged in PT infants, their ability to catch moving objects should be deficient. The purpose of the present experiment was to further investigate the ability of very preterm infants (VPT) to catch moving objects. Consequently, a number of sensory-motor parameters related to motor perception were examined.

\section{Methods}

Participants

The preterm infants were recruited at Uppsala University Hospital. They were all participants in an ongoing Longitudinal study of pre- and perinatal injuries to the VISual system (the LOVIS study). During a 4-year period (2004-2007), the parents to all surviving infants born before 32 weeks of gestational age (GA) in Uppsala County were asked to participate in the study. One hundred and thirteen infants out of 127 volunteered to participate in the LOVIS study. The infants included in the reach study did not differ on any major parameters compared with the rest of the LOVIS group, nor did they differ in prevalence of infants born small for gestational age, intraventricular hemorrhage (IVH), or bronchopulmonary dysplasia (BPD). However, none of the participating infants were diagnosed with PVL using ultrasound at 3-7 postnatal age and at 35 weeks of postmenstrual age. However, in the whole LOVIS population, only 7 infants were diagnosed with PVL in this manner. The selection procedure and a general description of the project can be found elsewhere (Strand Brodd et al. 2010).

A total of 47 preterm infants participated in the present experiment after invitation, 23 girls and 24 boys. The infants were studied as close to 8 months CA as possible (mean 37 week CA, SD 3.4 weeks). The average GA at birth was 28.6 weeks for the boys (range 23.4-31.9 weeks) and 28.3 weeks for the girls (range 24.6-31.9 weeks). Average birth weight for the boys was $1,217 \mathrm{~g}$ (range $559-1,717 \mathrm{~g}$ ) and for the girls $1,109 \mathrm{~g}$ (range $707-1,822 \mathrm{~g}$ ). Sixteen of these infants were diagnosed with retinopathy of prematurity (ROP), 4 with grade 3, 11 with grade 2 , and 1 with grade 1 . In the present experiment, the experimenter was blind to the medical history of the children.

Twenty FT infants constituting a control group were recruited by mail via public birth records, 12 boys and 8 girls. For the included infants, average GA at birth was 39.6 weeks (SD 1.8 weeks) and birth weight was 3,533 g (SD $417 \mathrm{~g}$ ). Mean age when visiting the laboratory was 35 weeks (SD 1.3 weeks).

Parents of all participants in this study provided written consent according to the guidelines specified in the 1964 Declaration of Helsinki. The study was approved by the Ethical Committee of the Medical faculty at Uppsala University (nr Ups 03-665).

Apparatus and stimuli

A small toy (radius maximum $2 \mathrm{~cm}$ ) attached to magnets moved on a vertical surface measuring $150 \times 150 \mathrm{~cm}$ (see 


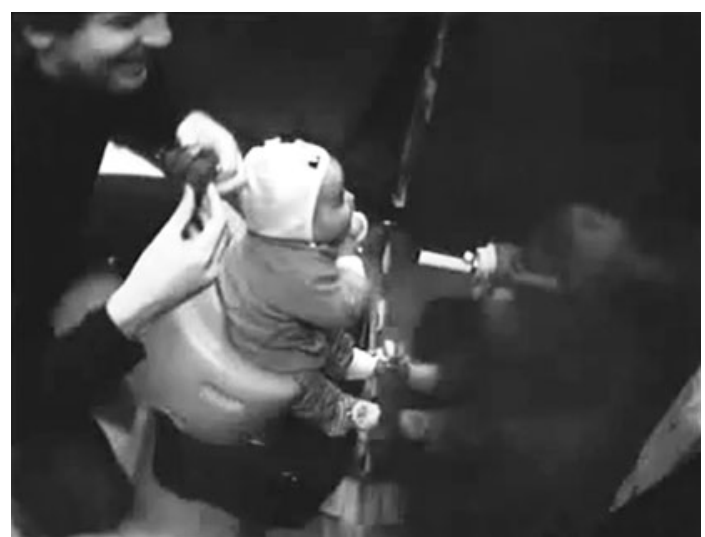

Fig. 1 Infant sitting in front of the display

Fig. 1). The motion was produced by another magnet situated on the back of the surface. When this magnet moved, the object in the front moved with it. A more detailed description of the vertical surface display is described elsewhere. (Gronqvist et al. 2006).

The infants sat in an infant chair that lent support to the lower torso (Bumbo Ltd, Gauteng, South Africa) facing the screen where the objects moved on the lower half of a circular trajectory with a radius of $35 \mathrm{~cm}$. The point where the object was closest to the subject was the lowest point on the semicircular trajectory. It was then right in front of the infant at breast height and clearly within reaching distance $(20 \mathrm{~cm})$. The $3 \mathrm{D}$ coordinates of the target motion and the movements of the hands of the infants were sampled at $240 \mathrm{~Hz}$ using five ProReflex cameras (Qualisys, Gothenburg, Sweden). The system uses passive reflective markers with the cameras placed above and on the sides of the subject. One marker was attached to the target and two markers to each hand of the infants (one at the base of the index finger and one at the base of the little finger to guarantee that at least one marker was visible when the hand was rotated). The Qualisys system has a pretrigger function that enables the experimenter to manually trigger the recording from before the actual triggering. In this study, each recording of data had a total duration of $4 \mathrm{~s}$, from $2 \mathrm{~s}$ before the manual triggering to $2 \mathrm{~s}$ after it. As soon as a reach was initiated, an experimenter pressed the pretrigger button. During the experiment, all trials were videotaped from above to allow the researchers to double check for reaches if necessary.

\section{Procedure}

Upon arriving to the laboratory, the parents were informed of the present experiment and signed a consent form. Two experimenters were present during the experiment: one who was responsible for the contact with the child and the parent/s and one who dealt with the equipment. When the infant had got used to the environment in the laboratory, Experimenter 1 placed two passive markers on each hand of the infant. The experimenter continued to interact with the infant showing some of the toys that were going to be used during the experiment. When the infant was in a good mood, he/she was placed in the infant chair in front of the display. The parent sat behind the infant. Experimenter 1 placed a toy on the display surface right above the magnet on the back of the screen and encouraged the infant to reach for it as it moved.

To get the infants used to the experimental situation, the session started with a warm-up phase. First, the toy moved vertically and stopped in front of the infant a couple of times and then it moved on a small pendulum path in front of the child. Sometimes it stopped right in front of the infant to entice them to reach. When the infant had tried to reach for the toy once or twice, the proper experiment began. The object then moved on the semicircular path. At each trial, the object moved from one side of the screen to the other and then back again and finally stopped out of reach of the child. Trials started randomly from the right or the left side. The velocity was modulated sinusoidally with the maximum velocity $(24 \mathrm{~cm} / \mathrm{s})$ at the lowest point of the trajectory. A full cycle had a duration of $15 \mathrm{~s}$. If the infant was efficient in grasping and reached successfully more than 3 times, the speed of the object was increased to a maximum velocity of $48 \mathrm{~cm} / \mathrm{s}$ (double that of the slower condition). If the child got tired or started to fuzz, a small break was made or the session was ended. As many reaches as possible were recorded up to 30 reaches. During the experiment, Experimenter 1 and/or the parent verbally encouraged the infant to reach. Throughout the experiment, Experimenter 2 sat out of sight and controlled the display movements and the pretriggering. The families got a small compensation for their participation. They could choose between a gift certificate in a book store or a toy store (value approximately $10 €$ ).

\section{Neuromotor examinations}

The present experiment was part of a larger study of very premature children (LOVIS). In addition to reaching, a battery of neuromotor examinations (NME) were performed by a physiotherapist and a neonatologist at 2 and 10 months CA. The infants' neurological status was examined by the neonatologist using a modified version of Touwen and Amiel-Tison's methods (Touwen 1978; Amiel-Tison and Grenier 1986; Touwen 1990). The physiotherapist used the instrument "Structured observation of Motor Behavior" (SOMP) that estimates both the infant's level of motor development and the quality of the achieved motor function level. At 2 months CA, there is a focus on spontaneous gross motor performance, contact, 
ability to follow an object, and the examiners face in supine, prone, assisted sitting, and assisted standing position. At 10 months $\mathrm{CA}$ the examination also includes sustained attention, social interaction, coordination, and short-term memory (Persson and Stromberg 1995). After the examinations, the neonatologist and the physiotherapist together evaluated the infants performance and expressed the result as $1=$ normal for $\mathrm{CA}, 2=$ suspicious of deviant for CA, or 3 = deviant for CA. A one-way ANOVA was used to compare the groups.

Data analysis

Each reach consisted of $4 \mathrm{~s}$ of data collection from the target and the reaching hand(s). A computer program was designed in MATLAB (P. Nyström department of Psychology, Uppsala University) where the trajectories of all markers were represented and plotted. The program made it possible to manually locate and select the data section where the infant was trying to reach for the object. The markers could get temporarily occluded by the infant's other hand, which resulted in occasional periods of missing data from the cameras. Such periods of missing data, where the trajectory of the marker could not be determined, were replaced with a linear interpolation of position. In total, only $0.96 \%$ of the data over all reaches were interpolated. All data were then filtered with a 7-sample-median filter to remove outlier values. This was done for $x, y$, and z-coordinates separately. To smoothen the data, a Butterworth low-pass filter at $10 \mathrm{~Hz}$ was applied. Thereafter, the velocity of all markers was calculated by comparing two consecutive $3 \mathrm{D}$ positions. The velocity profile was also smoothed by the Butterworth low-pass filter at $10 \mathrm{~Hz}$.

In order to analyze the data, movement units (MUs) of the reach were extracted. The MUs were based on the velocity profile, as in von Hofsten (von Hofsten 1991), with some modifications. A MU was defined as the region between two adjacent local minima that contained a velocity peak greater than $2.3 \mathrm{~cm} / \mathrm{s}$ above the minima. Thus, speed valleys marked the borderline of the unit (see Fig. 2). If the difference between the highest minima of one movement unit and the velocity peak of another was

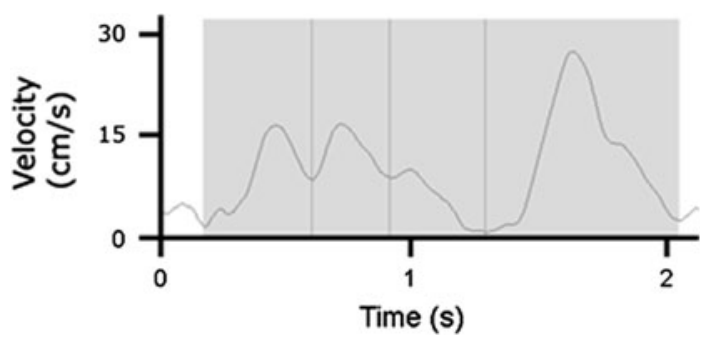

Fig. 2 Example of velocity profile with 4 MUs less than $8 \mathrm{~cm} / \mathrm{s}$, both units were merged into one single MU.

Two sets of data were selected: the (whole) reach and the transport unit (TU). A reach was defined as starting when the operating hand started to move consistently toward the target trajectory for at least $70 \mathrm{~mm}$ and ending when hand hit the target or began to move away from it in the case of a miss. The TU was defined as the longest MU of the hand.

The variables used for examining the reaching strategies and kinematics of the hand movements are presented in Table 1 and described below. The straightness of the movements was measured using relative length that is the ratio between the length of the trajectory of the hand and the straight line between the starting and ending coordinates (von Hofsten 1991). This gives a value of 1 if the hand moves in a straight line and otherwise a value over 1 . Max Jerk was calculated as the maximal change of acceleration $\left(\mathrm{mm} / \mathrm{s}^{3}\right)$. Point of Peak Velocity (PPV) is the percentage of the movement time from the start where the peak velocity occurred. For the whole reach, the number of MUs was calculated as well as the mean and maximal speed $(\mathrm{mm} / \mathrm{s})$.

To evaluate the infants' anticipation of the future positions of the moving object as they reached for it, the TU was used. Figure 3 illustrates how the angles were defined. A perfect predictive reach should be directed toward the point where the hand would meet the moving object at the end of the TU (B). The angle $\beta$ measured how far ahead the object moved during the TU and was defined as the angle $\mathrm{ACB}$, where $\mathrm{A}$ is the position of the object at the beginning of the TU, $\mathrm{C}$ is the position of the hand at the beginning of the TU, and $\mathrm{B}$ is the position of the object at the end of the TU. The angle $\alpha$ measured how far ahead the hand moved during the TU and was defined as the angle ACD, where D is the position of the hand at the end of the TU projected onto the approach plane ACB. The angle $\beta-\alpha$ then measures how the hand moves relative to the object. A positive aiming value indicates that the hand is lagging behind the object and perfect aiming results in zero.

Table 1 Variables used in the data analysis, decided by selection

\begin{tabular}{lll}
\hline & Reach & TU \\
\hline Relative length & $\mathrm{X}$ & $\mathrm{X}$ \\
Max jerk & $\mathrm{X}$ & $\mathrm{X}$ \\
Mean speed & $\mathrm{X}$ & $\mathrm{X}$ \\
Max speed & $\mathrm{X}$ & $\mathrm{X}$ \\
Aiming & & $\mathrm{X}$ \\
Movement unit & $\mathrm{X}$ & \\
Point of peak velocity & $\mathrm{X}$ & \\
Ratio 1:2 hands & $\mathrm{X}$ & \\
\hline
\end{tabular}




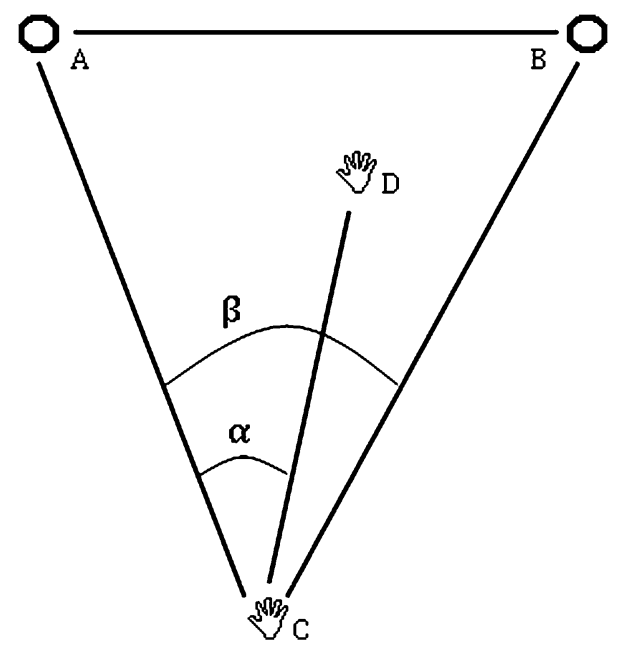

Fig. 3 The aiming measures $\alpha$ and $\beta$ shown schematically for a hypothetical TU. The line $A-B$ does not represent the actual path of the object, just the change in the angle to it

In total, 1,120 reaches were collected, 872 in the VPT group and 259 in the FT group. The following exclusion criteria were used to obtain a better signal to noise ratio: (a) if more than $20 \%$ of the whole reach/TU was interpolated and (b) if the length of the reach/TU was less than $70 \mathrm{~mm}$. For the VPT group, these criteria resulted in 25 excluded reaches and 89 excluded TUs; for the FT group, it resulted in 3 excluded reaches and 16 excluded TUs. The remaining reaches and TUs were averaged separately for each infant. Reaching data from infants are rarely complete since it is impossible to instruct the participants. In order to get a more complete data set, the slow and fast conditions were therefore collapsed as no differences were found between these conditions. Differences were obtained on 4 variables: relative length, mean speed, and MU for the reach and mean speed for the TU. In these cases, the ANOVA was made on the slow condition only. Hence, each infant got 1 data point per measure consisting of 2-30 reaches or TUs. The averages number of reaches performed was 17.8 .

In the cases where the infants used both their left and right hand when reaching, the hands were analyzed as two separate reaches (244 trials). The proportion of two hand reaches was calculated for each infant. A reach was defined as bimanual when both hands within one trial $(4 \mathrm{~s})$ fulfilled the criteria for a reach. The number of coupled reaches was also calculated and bimanual reaches were considered as coupled when the hands started to move within $0.5 \mathrm{~s}$ from one another.

The dependent variables in the analysis of the whole reach were relative length, number of MUs, speed, maximum jerk, PPV, and ratio between unimanual and bimanual reaches. The dependent variables of the analysis of the TU were the relative length, the aiming, speed, and maximum jerk. A successful reach was defined as the trials with target-hand contact.

Independent variables were gestational age, birth weight, and the results of neuromotor examinations (NME) at 2 and 10 months CA. First, the VPT group and the FT group were compared using an independent $t$ test. The VPT group was also divided into two subgroups: one with the infants born between 28 and 32 weeks of GA (VPT >27) and one with the infants born before 28 weeks of GA (VPT $<28$ ). They were then compared with the FT group using a one-way ANOVA. The VPT infants were also divided into subgroups according to their birth weight: low birth weight under 1,000 $\mathrm{g}(\mathrm{LBW}<1,000 \mathrm{~g}$ ) and low birth weight (LBW $>1,000 \mathrm{~g})=1,000-2,499 \mathrm{~g}$; weight groups were compared in a one-way ANOVA. The correlation between gestational age and birth weight was 0.83 .

\section{Results}

Of the 47 VPT infants, 3 did not reach resulting in 44 infants to be analyzed. There were $20 \mathrm{FT}$ infants of whom 6 did not make any reaches resulting in 14 infants to be analyzed.

Overall, FT as well as VPT infants reached predictively and anticipated the upcoming position of the moving object. The hand was on the average 5.8 degrees ahead of the object at the end of the $\mathrm{TU}$, which corresponds to between 2 and $6 \mathrm{~cm}$. The reaches consisted of 2-3 movement units $(\mathrm{M}=2.33, \mathrm{SD}=0.51)$. PPV was on average at $52 \%(\mathrm{SD}=6)$. The infants encountered the object in $38 \%$ of the reaches for both the VPT and the FT groups.

All the different kinematic variables were analyzed with respect to gestational age, birth weight, and the different aspects of the neuromotor examination. Only the significant effects are reported as follows.

\section{Gestational age}

\section{Reach}

The VPT group used two hands in $20.8 \%$ (SD $=14.4$ ) of their reaches, whereas FT group used it in $12.0 \%$ $(\mathrm{SD}=7.5)(\mathrm{t}(56)=2.189, P<0.05)$. The VPT infants $(\mathrm{M}=61 \%)$ used proportionally more coupled bimanual reaches than the FT infants $(M=40 \%) \quad \chi^{2} \quad(2$, $N=244)=5.561, P<0.05$.

A one-way ANOVA showed a difference in overall proportion of bimanual reaches (coupled and uncoupled) when dividing the VPT group into the VPT $>27$ and VPT $<28$ subgroups $(\mathrm{F}(2,55)=4.012, P<0.05)$. A Tukey test revealed that the VPT $<28$ group $(\mathrm{M}=25.6 \%, \mathrm{SD}=14)$ used more bimanual reaches (coupled and uncoupled) than 
the FT group $(\mathrm{M}=12 \%, \mathrm{SD}=7.5, P<0.05)$. The VPT $>27$ group $(\mathrm{M}=18.4 \%, \mathrm{SD}=14.3)$ did not differ significantly from the other groups.

\section{$T U$}

The relative length of the TU differed between the groups, $\mathrm{F}(2,55)=5.524, P<0.01$. Post hoc test showed that the VPT $<28$ group $(\mathrm{M}=1.26, \mathrm{SD}=0.10)$ had a less straight movement than both the VPT $>27(\mathrm{M}=1.20, \mathrm{SD}=0.07$, $P<0.05)$ and FT $(\mathrm{M}=1.18, \quad \mathrm{SD}=0.05, \quad P<0.01)$ infants. This is the only TU variable for which there is a difference with respect to GA.

Birth weight

Reach

Birth weight gave similar results as gestational age. Proportion of bimanual reaches (coupled and uncoupled) differed, $\mathrm{F}(2,55)=5.194, P=0.009$. The LBW $<1,000 \mathrm{~g}$ $(\mathrm{M}=26.6 \%)$ infants more often used 2 hands compared with the FT $(\mathrm{M}=12 \%, P=0.007)$ and had a tendency to use more bimanual reaches (coupled and uncoupled) than the LBW $>1,000 \mathrm{~g}$ infants $(\mathrm{M}=17.5 \%, P=0.066)$.

Birth weight also had an effect on the maximum jerk of the reach, $\mathrm{F}(2,55)=4.281, P=0.019$. The LBW $<1,000 \mathrm{~g}(\mathrm{M}=20.88)$ infants had higher maximum jerk than the LBW $>1,000 \mathrm{~g}(\mathrm{M}=13.42, P=0.031)$ and FT $(\mathrm{M}=12.41, P=0.037)$ infants.

\section{$T U$}

The relative length of the $\mathrm{TU}$ also differed between the groups, $\mathrm{F}(2,55)=3.171, P=0.050$. Post hoc test showed

Table 2 Distribution of neurological examinations (NME)

\begin{tabular}{lllc}
\hline & NME 1 & NME 2 & NME 3 \\
\hline 2 months & 23 & 9 & 12 \\
10 months & 26 & 8 & 9 \\
\hline
\end{tabular}

NME 1 normal for corrected age, NME 2 is suspicious of deviant for corrected age, and NME 3 is deviant for corrected age that the FT group $(\mathrm{M}=1.176)$ had a straighter TU compared with the LBW $<1,000 \mathrm{~g}$ group $(\mathrm{M}=1.244$, $P=0.044)$.

Neuromotor examinations

Distribution of the infants' results in the NME is shown in Table 2. The larger proportion of the VPT group is assessed as normal for their corrected age. Some infants shift groups between 2 and 10 months CA. Five of the infants who were deviant at 2 months were also deviant at 10 months. Of the remaining seven infants, three were assessed as normal, three were assessed as suspicious of deviancy, and one was not assessed at 10 months. Two infants assessed as normal and two assessed as suspicious of deviant at 2 months were added to the deviant group at 10 months.

\section{2 months}

The infants assessed to be in group 3 (lowest performance), at 2 months CA, differed from other groups when reaching. Their maximum speed for the reach was higher than for the other groups, $\mathrm{F}(3,54)=5.136, P<0.01$ (Table 3). Maximum speed for group 3 was higher than for group 2 $(P<0.01)$, group $1(P<0.05)$, and the control group $(P<0.05)$. The maximum acceleration also differed between the groups, $\mathrm{F}(3,54)=2.856, P<0.05$. The post hoc test revealed differences between groups 2 and 3 $(P<0.05)$ (Table 3).

As for the reach, the maximum speed of the TU differed between the groups, $\mathrm{F}(3,54)=3.991, P<0.05$. Maximum speed for group 3 was higher than for group $2(P<0.05)$ and the control group $(P<0.05)$. The maximum acceleration of the TU had the same pattern as the maximum speed, $\mathrm{F}(3,54)=4.930, P<0.001$. Group 3 had higher acceleration than group $2(P<0.01)$ and the controls $(P<0.01)$. Group assignment also had an overall effect on maximum jerk of the TU, $\mathrm{F}(2,55)=3.515, P<0.05$; however, no significant effects were found between the single categories. The difference between groups 2 and 3 was, however, marginally significant, $P=0.057$ (Table 3 ).
Table 3 Reaching results comparing reaching characteristics by results on neuromotor examinations at 2 months (CA) and FT controls

Group mean (SD)

\begin{tabular}{lcccc}
\hline & NME 1 & NME 2 & NME 3 & FT \\
\hline Reach & & & & \\
Max speed (mm/s) & $503.7(91.2)$ & $444.7(93.9)$ & $636.1(170.2)$ & $498.4(121.5)$ \\
Mean acceleration $\left(\mathrm{mm} / \mathrm{s}^{2}\right)$ & $31.2(9.3)$ & $25.9(6.9)$ & $39.5(17.5)$ & $28.9(10.5)$ \\
TU & & & & \\
Max speed $(\mathrm{mm} / \mathrm{s})$ & $462.2(86.0)$ & $421.9(93.0)$ & $569.4(150.1)$ & $451.9(104.9)$ \\
Max acceleration $\left(\mathrm{mm} / \mathrm{s}^{2}\right)$ & $21.4(5.05)$ & $17.9(4.6)$ & $27.4(10.7)$ & $18.8(5.0)$ \\
Max jerk $\left(\mathrm{mm} / \mathrm{s}^{3}\right)$ & $9.00(4.17)$ & $5.89(1.60)$ & $11.42(8.24)$ & $8.44(4.99)$ \\
\hline
\end{tabular}


Table 4 Reaching results (means and SD) comparing reaching characteristics and results on the neuromotor examination at 10 months (CA) and the results of the FT controls

\begin{tabular}{lrrrrrrrr}
\hline & NME 1 & NME 2 & & NME 3 & \multicolumn{2}{l}{ FT } \\
\hline TU & & & & & & & & \\
Aiming & -5.58 & 8.20 & -0.29 & 4.53 & -11.02 & 7.44 & -4.79 & 5.31 \\
Alpha & 36.02 & 9.61 & 26.31 & 6.47 & 40.98 & 11.41 & 36.94 & 12.13 \\
\hline
\end{tabular}

\section{0 months}

The groups from neurological assessments at 10 months CA did not differ on any kinematic variables; however, differences were found for aiming, $\mathrm{F}(3,53)=3.107$, $P<0.05$ (Table 4). Post hoc test shows that infants in group 3 aimed further ahead of the target $(\alpha-\beta)$ than those in group $2(P<0.05)$. How far ahead of the object position at the beginning of the movement $(\alpha)$ the reach was aimed also differed between the groups, $\mathrm{F}(3,53)=2.787, P<0.05$; group 3 has a larger $\alpha$ angle than group $2(P<0.05)$.

\section{Discussion}

This study investigated how reaching for moving objects was affected by preterm birth. It was found that the VPT and the FT infants were equally successful in getting to the goal and touching the object. The VPT group, however, made twice as many bimanual reaches as FT infants and used a more curved and jerky path (more change in acceleration) when reaching.

What could be the reason for using two hands while catching? It seems to be a more robust strategy to catch a moving object, for example adults use two hands for more challenging tasks such as catching a fly ball. Earlier research on FT infants has shown that the use of uni- and bimanual strategies when reaching for stationary as well as moving objects fluctuates during the first year (Rochat 1992; Corbetta and Thelen 1996; van Hof et al. 2005). When reaching for moving objects, a cross-sectional study showed that infants used more bimanual reaches (as defined in this paper) at 6 and 10 months and more unimanual reaches at 8 months (Fagard et al. 2009). Is the VPT group simply adjusting to a situation they perceive as challenging or is it a developmental delay? Non-self-sitting FT infants use more bimanual reaching than self-sitting infants (Rochat 1992). The VPT $<28$ weeks and LBW $<1,000 \mathrm{~g}$ infants used the highest amounts of bimanual reaches, namely in $1 / 4$ of the trials. A common feature of these groups is the fact that they are small at birth that earlier has been reported to delay onset of self-sitting (Marin Gabriel et al. 2009). Using both hands in a symmetrical way helps the infants to maximize stability and precision. Even noncoupled asymmetrical reaches can have a balancing effect on point of gravity. In the present study, the lower torso of the infants was supported by the Bumbo seat. Under such condition, their reaching should not have been affected by stabilizing concerns. The VPT infants could, however, have learnt that bimanual reaching is a safer strategy under any condition. The fact that the object is moving puts certain demands on the reach and its timing. Using two hands maximizes reaching range both in space and in time. It seems possible that this increased bimanual strategy reflects some general delay or immaturity of the motor system.

The relative length of the TU differed between the groups. For the VPT $<28$-week infants, TU was larger than for the VPT $>27$ weeks and FT infants. The more circuitous trajectories were not a result of an inability to predict the motion of the object, because the different groups of infants predicted the upcoming positions of the objects equally well and even aimed slightly ahead of TU of the object. This indicates that the VPT group uses less efficient procedures while reaching rather than an inferior planning.

The VPT infants assessed to have a deviant NME at 2 months CA showed a jerkier movement profile (more change in acceleration) and a higher maximum speed as they reached for moving objects. These results are different from earlier research showing lower mean velocity for VPT infants (Toledo and Tudella 2008). However, Toledo and Tudella (2008) used stationary objects and studied low-risk infants born 32-36 weeks of GA, whereas in the present study the increased reaching velocity was found for infants with deviant neuromotor development. Moving objects also pose more severe time constraints compared with stationary objects. The VPT infants assessed as deviant at 10 months aimed further ahead of the object compared with the infants suspicious of deviance. These results indicate some kind of compensatory behavior. Forsström and von Hofsten (1982) studied reaching for moving objects in children with minor brain dysfunctions (MBD) and found that their reaching was less well organized than the reaching of typically developed children. The aiming, however, was more ahead of the object than in typically developed children, which was interpreted as a compensatory strategy for getting more time to approach of the object.

The neurological assessment investigates somewhat different capacities at the different ages. At 2 months, it focuses on basic motor skills, concentration, and awareness, whereas the assessment at 10 months also considers cognitive and coordinative components as the tasks are more complicated and requires more intentionally driven behavior and interaction with the investigator. The infants assessed as deviant in NME at 2 months compensated by 
moving faster to catch up while infants assessed as deviant in NME at 10 months aim ahead to get more time for the reach. Only 5 of the infants assessed as clearly deviant at 2 months had the same assessment at 10 months. If the infants were deviant at 2 but not at 10 months, they had caught up between these ages according to the neurological assessment which was the case for 8 of the infants. But as a group they still showed somewhat different reaching pattern at 8 months. The VPT infants who were deviant at 10 months included 2 infants assessed as normal and 2 assessed as suspiciously deviant at 2 months CA. These infants had then come to lag behind in development of motor performance.

None of the infants were diagnosed with PVL using ultrasound of the brain during the neonatal period that possibly is an underestimation. Earlier research has shown that ultrasound has severe limitations in detecting the more common diffuse noncystic white matter injuries (Inder et al. 2003). The differences in relative length of the TU show an ineffective way of programming the reach and could be strategies compensating for reduced capacity to interpret the future position of the object. This could be an effect of developmental problems in the magno-cellular pathways. It is also reasonable to believe that the poor reaching strategies in the groups with deviant NME reflect a higher proportion of diffuse PVL in this group. These relationships will be further evaluated in the long-term follow-up as the VPT group will have MRT scans at 30-42 months of age.

In conclusion, looking at the reach as a goal-directed action, the VPT group is as efficient as the FT group as they hit the object equally often. They also aimed equally well and used the same number of movement units. However, the VPT group is not equally skilled in all respects. They seem to require more effort when reaching, possibly reflecting developmental lag in coordination and execution. VPT infants with deviant NME displayed additional compensatory behavior in terms of aiming strategies and faster reaches.

The fact that the VPT infants in the present study had 2-4 months more extrauterine experience than the FT infants did not improve reaching performance. If there are any advantages to being born prematurely, it is overshadowed by the higher risk of disturbances to brain development for this group, e.g., hypoxic lesions to the afferent pathways in the white matter, i.e., (PVL) (Volpe 2001).

Acknowledgments We thank all parents and infants making this work possible. Thanks to staff at the department of women's and children's health, especially Kristina Persson, Bo Strömberg and Uwe Ewald, department of neuroscience; Gerd Holmström, department of psychology; Kerstin Rosander, Pär Nyström, and the babylab team, Uppsala University. This work is supported by grants to $\mathrm{CvH}$ from the Swedish Research council (VR421-2004-1883) and grants from the
Norwegian Directorate for Children, Youth and Family Affairs (06/ 34707).

Open Access This article is distributed under the terms of the Creative Commons Attribution Noncommercial License which permits any noncommercial use, distribution, and reproduction in any medium, provided the original author(s) and source are credited.

\section{References}

Amiel-Tison C, Grenier A (1986) Neurological assessment during the first year of life. Oxford University Press, New York

Atkinson J (2000) The developing visual brain. Oxford University Press, Oxford, New York

Corbetta D, Thelen E (1996) The developmental origins of bimanual coordination: a dynamic perspective. J Exp Psychol Hum Percept Perform 22:502-522

Fagard J, Spelke E, von Hofsten C (2009) Reaching and grasping a moving object in 6-, 8-, and 10-month-old infants: laterality and performance. Infant Behav Dev 32:137-146

Fallang B, Saugstad OD, Grogaard J, Hadders-Algra M (2003) Kinematic quality of reaching movements in preterm infants. Pediatr Res 53:836-842

Fallang B, Oien I, Hellem E, Saugstad OD, Hadders-Algra M (2005) Quality of reaching and postural control in young preterm infants is related to neuromotor outcome at 6 years. Pediatr Res 58:347-353

Forsström A, von Hofsten C (1982) Visually directed reaching of children with motor impairments. Dev Med Child Neurol 24:653-661

Goodale MA, Milner AD (1992) Separate visual pathways for perception and action. Trends Neurosci 15:20-25

Gronqvist H, Gredeback G, Hofsten C (2006) Developmental asymmetries between horizontal and vertical tracking. Vision Res 46:1754-1761

Hoon AH Jr, Lawrie WT Jr, Melhem ER, Reinhardt EM, Van Zijl PC, Solaiyappan M, Jiang H, Johnston MV, Mori S (2002) Diffusion tensor imaging of periventricular leukomalacia shows affected sensory cortex white matter pathways. Neurology 59:752-756

Inder TE, Anderson NJ, Spencer C, Wells S, Volpe JJ (2003) White matter injury in the premature infant: a comparison between serial cranial sonographic and MR findings at term. AJNR Am J Neuroradiol 24:805-809

Jacobson L, Ek U, Fernell E, Flodmark O, Broberger U (1996) Visual impairment in preterm children with periventricular leukomalacia-visual, cognitive and neuropaediatric characteristics related to cerebral imaging. Dev Med Child Neurol 38:724-735

Kayed NS, Van der Meer AL (2009) A longitudinal study of prospective control in catching by full-term and preterm infants. Exp Brain Res 194:245-258

Marin Gabriel MA, Pallas Alonso CR, De La Cruz Bertolo J, Caserio Carbonero S, Lopez Maestro M, Moral Pumarega M, Alonso Diaz C, Lora Pablos D (2009) Age of sitting unsupported and independent walking in very low birth weight preterm infants with normal motor development at 2 years. Acta Paediatr 98:1815-1821

Persson K, Stromberg B (1995) Structured observation of motor performance (SOMP-I) applied to preterm and full term infants who needed neonatal intensive care. A cross-sectional analysis of progress and quality of motor performance at ages 0-10 months. Early Hum Dev 43:205-224

Rochat P (1992) Self-Sitting and Reaching in 5- to 8-month-old infants: the impact of posture and its development on early eyehand coordination. J Mot Behav 24:210-220 
Strand Brodd K, Ewald U, Grönqvist H, Holmström G, Strömberg B, Von Hofsten C, Rosander K (2010) LOVIS-Preterm born infants show delayed development of Smooth Pursuit Eye Movements in a LOngitudinal multidisiplinary study of VISuomotor capacity. Manuscript

Toledo AM, Tudella E (2008) The development of reaching behavior in low-risk preterm infants. Infant Behav Dev 31:398-407

Touwen BCL (1978) Liber Amicorum Ronald MacKeith clinics in developmental medicine

Touwen BC (1990) Variability and stereotypy of spontaneous motility as a predictor of neurological development of preterm infants. Dev Med Child Neurol 32:501-508 van Hof P, van der Kamp J, Caljouw SR, Savelsbergh GJP (2005) The confluence of intrinsic and extrinsic constraints on 3- to 9-month-old infants' catching behaviour. Infant Behav Dev 28:179-193

Volpe JJ (2001) Neurobiology of periventricular leukomalacia in the premature infant. Pediatr Res 50:553-562

von Hofsten C (1980) Predictive reaching for moving objects by human infants. J Exp Child Psychol 30:369-382

von Hofsten C (1983) Catching skills in infancy. J Exp Psychol Hum Percept Perform 9:75-85

von Hofsten C (1991) Structuring of early reaching movements: a longitudinal study. J Mot Behav 23:280-292 